\title{
INTRODUCTION OF A UNIVERSAL P3O0 BRAIN-COMPUTER INTERFACE COMMUNICATION SYSTEM
}

\author{
Andreas Pinegger ${ }^{1}$, Selina Wriessnegger ${ }^{1}$, Gernot Müller-Putz $^{1}$ \\ ${ }^{1}$ Institute for Knowledge Discovery, Graz University of Technology , Austria \\ a.pinegger@tugraz.at
}

\begin{abstract}
We developed a new P300-based BCI communication system. The design is tripartite: One part operates as a universal data acquisition unit, which allows to easily use different data acquisition devices. The second part is a rapid prototyping platform based on Matlab/Simulink ${ }^{\circledR}$ for data processing, which can be modified in an easy way. The last part is a graphical user interface, which also acts as main controller. Every single part is state-of-the-art designed and implemented. Connected together they are a very powerful tool not only for scientists and research issues, but also for non-expert users.
\end{abstract}

Keywords: P300, BCI, user-centered-design, famous faces

\section{Introduction}

A brain-computer interface (BCI) is an interface that connects a human brain directly with a computer. It recognizes mentally induced changes of brain signals, in our case the electroencephalogram (EEG) and forms a control signal.

There are different kinds of brain activity patterns which can be used for a BCI. One of them, the P300 phenomenon, is an event-related potential(ERP), triggered by unexpected, rare, or particularly informative stimuli. It is described as a positive peak visible in the EEG approximately $300 \mathrm{~ms}$ after the stimuli.

Donchin and colleagues presented in [1] the first P300based BCI, also called P300 speller, which permits to spell words. A $6 \times 6$ matrix filled with letters and symbols is presented to the user, and entire columns or rows are flashed one after the other in random order. When the column/row containing the desired letter is flashed, a P300 is elicited.

If the stimulus is more complex than just a flash, other ERPs are generated too. Kaufmann and colleges showed in [2] that the use of famous face images as stimuli cause two further negative deflections, the N170 and N400f, and that these can be additionally used for classification. This modification improves the classification rates significantly.

In this work we present our newly developed universal P300-famous faces speller, see Fig.1, based on the ideas of Donchin and Kaufmann. With this BCI the user is able to spell, to control a multimedia player, or to browse the Internet.

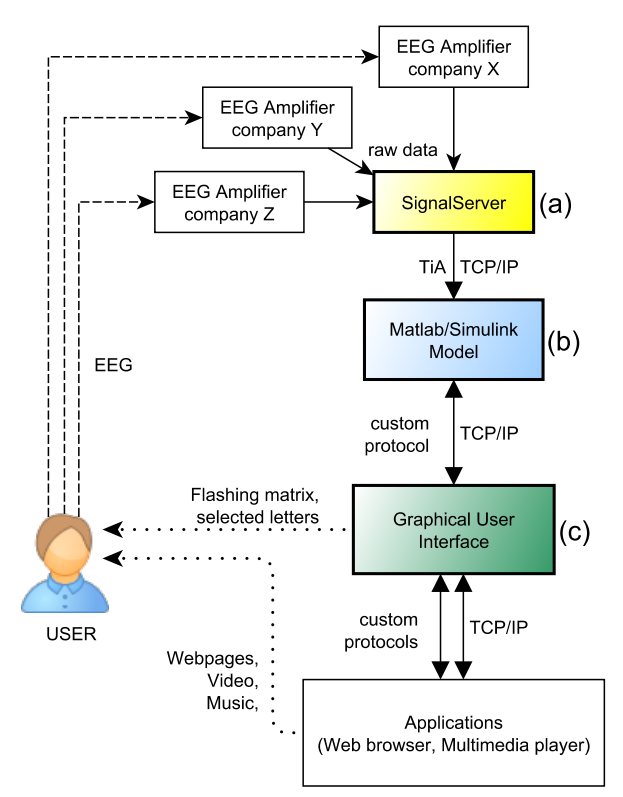

Figure 1: Design sketch of the universal P300 BCI communication system.

\section{Methods}

Data acquisition:

For data acquisition the TOBI SignalServer software [3] is used, see Fig. 1a). The big advantage of this software is that it can handle many different data acquisition devices [4].

\section{Paradigm:}

The paradigm is inspired by the work of Donchin [1] which has been described before. The only differences are (i) the matrix has a fixed size of $6 \times 6$ just during the calibration and can have an $n \times 6$ (with $n=6 \ldots 14$ ) size afterwards, and (ii) the intensification of the rows/columns is done with famous faces instead of flashing them.

\section{Data processing:}

Matlab/Simulink ${ }^{\circledR}$ (The MathWorks, USA) is used for data processing, see Fig. 1b). The processing itself is mainly based on results found in [5] with some deviations.

For each channel, $800 \mathrm{~ms}$ segments of data following each intensification are extracted. Afterwards a baseline correction with $200 \mathrm{~ms}$ pre-stimulus data is performed. The segments are then moving average filtered and decimated by equivalent values. The resulting data segments are concatenated by channel for each intensification (highlighting 


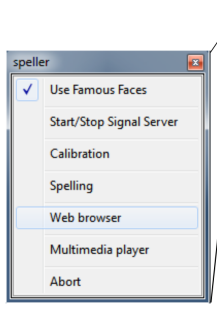

(a)

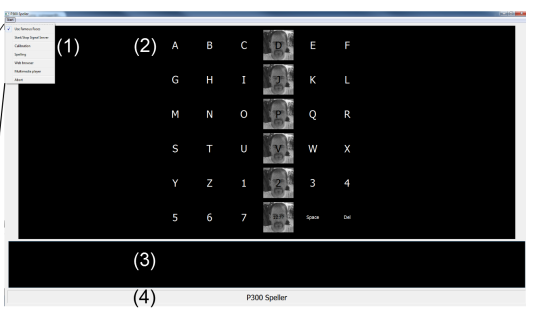

(b)
Figure 2: Screen-shot of a) the user menu and b) the P300 user interface. The different areas are (1) the menu, (2) the P300 matrix, (3) the text field, and (4) the status bar. Normally famous faces are used which are not shown in this figure due to print license.

of a row or column), creating a single feature vector. For each stimulus one feature vector per sequence (all rows and columns highlighted once) is generated and averaged over all sequences (maximum 15 per selection).

In training mode this is done for ten letters and afterwards a classifier is trained using stepwise linear discriminant analysis (SWLDA) [6]. To get the best trade-off between speed and accuracy the lowest number of sequences is calculated with a leave-one-out cross-validation (LOOCV) and successive adding of sequences to the test set. However, the practically best number of sequences is determined afterwards as described in [7].

In spelling mode the calculated classifier and the determined best number of sequences will automatically be used.

\section{Results}

We developed a P300 BCI system which is based on three main components: The TOBI SignalServer for data acquisition and distribution, a Matlab/Simulink ${ }^{\circledR}$ model for data processing and an in $\mathrm{C}++$ implemented graphical user interface and paradigm control, see Fig. 2. Within the application it is possible to start and stop the data acquisition software (SignalServer). Once the Matlab/Simulink ${ }^{\circledR}$ model is running in the background the training or spelling session can be started just by one click. Also the image (preferred famous face) for highlighting can be selected freely just by three mouse clicks.

The communication with Matlab/Simulink ${ }^{\circledR}$ is done via a network (TCP/IP) connection. Two further TCP/IP connections to control other applications are already implemented: One to control a web browser and one to control a multimedia player. To fulfill the needs of these two applications the size of the matrix adapts automatically, as described before.

\section{Discussion}

This work introduces a state-of-the-art P300 BCI system based on famous faces. It was designed and developed for the EC founded project BackHome (www.backhome- fp7.eu). It should be easy and intuitive in use with simultaneous consideration of the latest research results. To achieve this, it combines the universal data acquisition interface idea from [4] with the improvements of Kaufmann and colleges [2] and [7] showing letters highlight as famous faces and a user-centered, easy-to-use graphical user interface design. Regarding the data processing part the design is focused on best on-line performance as it is described in [5] and [7].

From a scientific perspective the use of Matlab/Simulink ${ }^{\circledR}$ as data processing and partly control software allows scientific users to easily implement and test new algorithms or paradigm control structures to address research questions. The two implemented interfaces to external applications (multimedia player and web browser), which are still under development, round off the universal applicability of this system.

\section{Acknowledgement}

Supported by the FP7 research project BackHome (No. 288566). This paper only reflects the author's views and funding agencies are not liable for any use that may be made of the information contained herein.

\section{Bibliography}

[1] E. Donchin, K. Spencer, and R. Wijesinghe, "The mental prosthesis: Assessing the speed of a p300-based brain- computer interface," IEEE Transactions on Rehabilitation Engineering, vol. 8, no. 2, pp. 174-179, 2000.

[2] T. Kaufmann et al., "Flashing characters with famous faces improves erp-based brain-computer interface performance," Journal of Neural Engineering, vol. 8, no. 5, 2011.

[3] C. Breitwieser et al., "Proposing a standardized protocol for raw biosignal transmission," IEEE Transactions on Biomedical Engineering, vol. 59, no. 3, pp. 852859, 2012.

[4] G. R. Müller-Putz et al., "Tools for brain-computer interaction: a general concept for a hybrid bci (hbci)," Frontiers in Neuroinformatics, vol. 5, no. 30, 2011.

[5] D. Krusienski et al., "Toward enhanced p300 speller performance," Journal of Neuroscience Methods, vol. 167 , no. 1 , pp. $15-21,2008$.

[6] D. J. Krusienski et al., "A comparison of classification techniques for the p300 speller," Journal of Neural Engineering, vol. 3, no. 4, p. 299, 2006.

[7] T. Kaufmann et al., "Spelling is just a click away - a user-centered brain-computer interface including autocalibration and predictive text entry," Frontiers in Neuroscience, vol. 6, no. 72, 2012. 